\title{
Observer Based Output Feedback Tracking Control of Dynamically Positioned Surface Vessels
}

\author{
Baris Bidikli, Enver Tatlicioglu, and Erkan Zergeroglu
}

\begin{abstract}
This work concentrates on tracking control of dynamically positioned surface vessels where only position and orientation measurements are available. Specifically, in order to remove the velocity measurement dependency of the control formulation, we designed a nonlinear, model-free observer which enables the observer-controller couple to achieve asymptotic tracking. Stability of the closed-loop system is ensured by Lyapunov-based arguments. Simulation studies are also presented to illustrate the effectiveness of the proposed method.
\end{abstract}

\section{INTRODUCTION}

Control of marine vehicles, especially slowly moving surface vessels, is extremely important in marine industry. Operations where a smooth, slow trajectory needs to be tracked, like towing platforms, laying cables to the sea bottom, and most of the operations related to the offshore oil industry requires adequate controllers. As a result, the development of automatic ship control systems have attracted the attention of researchers over the past decade.

A dynamically positioned surface vessel is a fully actuated ship system where its three degrees-of-freedom are controlled via thrusters and propellers fore and aft of the ship [1], [2]. Earliest control systems for dynamically positioned ships were mostly designed after linearizing the system dynamic equation about a set of pre-specified yaw angles [3]. This procedure enables the application of linear control methods along with gain scheduling techniques. For example, early ship control systems used proportional integral derivative controllers in cascade with a low-pass filter [4]. Later, linear optimal control laws in conjunction with Kalman filtering techniques were proposed in [5], [6], [7]. To overcome the problems inherited by linearization, several control algorithms that take the nonlinear ship dynamics into account have also been proposed [8], [9]. In [8], a class of nonlinear proportional derivative control laws for position regulation were developed; however, their robustness against parametric uncertainties cannot be guaranteed. A robust nonlinear control law using singular perturbation theory that accounts for parametric uncertainties and external disturbances was presented in [9].

Some past research has focused on designing control schemes that do not require velocity measurements for

B. Bidikli is with the Department of Electrical \& Electronics Engineering, Celal Bayar University, Manisa, Turkey barisbidikli@cbu.edu.tr E. Tatlicioglu is with the Department of Electrical \& Electronics Engineering, Izmir Institute of Technology, 35430, Izmir, Turkey envertatliciogluaiyte.edu.tr

E. Zergeroglu is with the Department of Computer Engineering, Gebze Institute of Technology, 41400, Gebze, Kocaeli, Turkey ezergerabilmuh. gyte. edu.tr surface vessels that contain only position sensors. Motivated by this, in [3], Fossen and Grøvlen presented the design of a nonlinear output feedback controller using an observer backstepping method. Specifically, a nonlinear, model-based observer-controller couple was used to eliminate the need for velocity measurements while achieving global exponential position tracking. In [10], a velocity surrogate filter-based approach has been applied for adaptive output feedback control of surface vessels. The proposed method achieved global asymptotic tracking despite the lack of velocity measurements and uncertain system dynamics. Recently, in [11], Wondergem et al. proposed an observer based output feedback tracking controller for fully actuated ships. The proposed controller achieved semi-global exponential stability provided the exact knowledge of the system parameters are available for control development.

The main aim of this work is the design of an output feedback tracking controller for dynamically positioned surface vessels. Our starting point for the proposed approach is the fact that the nonlinear ship model can be arranged in a form similar to the well-known rigid-link, robot manipulator dynamic model. Using this fact, to compensate for the lack of velocity measurements, we propose a new model-free observer in conjunction with a desired ship model based controller formulation. The observer-controller couple ensures semi-global asymptotic position tracking for the nonlinear surface vessel dynamics using only position measurements.

The rest of the paper is organized in the following manner. Section II describes the mathematical model for the dynamically positioned ship system along with its corresponding properties. The control objective and problem formulation are presented in Section III, while the design and stability analysis are presented in Section IV. Numerical simulation results illustrating the performance of the proposed observercontroller scheme are given in Section V. Section VI contains concluding remarks.

\section{SYSTEM MODEL AND PROPERTIES}

The mathematical model for a dynamically positioned ship is represented by [1]

$$
\begin{aligned}
M \dot{v}+D v & =\tau \\
\dot{\eta} & =R(\psi) v
\end{aligned}
$$

where $\eta(t), v(t) \in \mathbb{R}^{3}$ represent the position and the velocity of the ship, respectively, $\tau(t) \in \mathbb{R}^{3}$ represents the control input torque, $M \in \mathbb{R}^{3 \times 3}$ is the constant, positivedefinite, symmetric, inertia matrix, $D \in \mathbb{R}^{3 \times 3}$ is the constant damping matrix, and $R(\psi) \in S O(3)$ is the rotation matrix 
between the earth and the body-fixed coordinate frames. In (1) and (2), $\eta(t)=[x(t), y(t), \psi(t)]^{T}$ where $x(t)$, $y(t) \in \mathbb{R}$ represent the translational position, and $\psi(t) \in \mathbb{R}$ is the yaw angle of the ship. The structure of the system matrices are given as

$$
M=\left[\begin{array}{lll}
m_{11} & 0 & 0 \\
0 & m_{22} & m_{23} \\
0 & m_{23} & m_{33}
\end{array}\right], D=\left[\begin{array}{lll}
d_{11} & 0 & 0 \\
0 & d_{22} & d_{23} \\
0 & d_{32} & d_{33}
\end{array}\right]_{3}
$$

where their entries are constants, and the rotation matrix $R(\psi)$ has the form

$$
R(\psi)=\left[\begin{array}{lll}
c_{\psi} & -s_{\psi} & 0 \\
s_{\psi} & c_{\psi} & 0 \\
0 & 0 & 1
\end{array}\right]
$$

where $c_{\psi}$ and $s_{\psi}$ represent $\cos (\psi)$ and $\sin (\psi)$, respectively. After substituting (2) and its time derivative into (1), the mathematical model of the ship can be written in a compact form as

$$
J \ddot{\eta}+C \dot{\eta}+F \dot{\eta}=\tau^{*}
$$

where $J(\eta), C(\eta, \dot{\eta}), F(\eta) \in \mathbb{R}^{3 \times 3}$ are dynamic terms ${ }^{1}$, $\tau^{*}(t) \in \mathbb{R}^{3}$ is the control input torque, and are defined as [10]

$$
\begin{aligned}
J & \triangleq R M R^{T}, C \triangleq R M \dot{R}^{T} \\
F & \triangleq R D R^{T}, \tau^{*} \triangleq R \tau
\end{aligned}
$$

where the fact that $R^{-1}=R^{T}$ was utilized. The dynamic model given by (5) satisfies following properties.

Property 1: The inertia matrix $J(\eta)$ is symmetric, positive-definite, and satisfies the following bounds

$$
\begin{aligned}
m_{l} I_{3} & \leq J \leq m_{u} I_{3} \\
\frac{1}{m_{u}} I_{3} & \leq J^{-1} \leq \frac{1}{m_{l}} I_{3}
\end{aligned}
$$

where $m_{l}, m_{u} \in \mathbb{R}$ are positive bounding constants, and $I_{3} \in \mathbb{R}^{3 \times 3}$ is the standard identity matrix.

Property 2: The dynamic terms $J(\eta)$ and $C(\eta, \dot{\eta})$ satisfy the skew-symmetric relationship [12]

$$
\varphi^{T}\left(\frac{1}{2} \dot{J}-C\right) \varphi=0 \forall \varphi \in \mathbb{R}^{3} .
$$

Property 3: The dynamic term $C(\eta, \dot{\eta})$ satisfies the relationship [12]

$$
C(\varphi, \phi) \kappa=C(\varphi, \kappa) \phi \forall \varphi, \phi, \kappa \in \mathbb{R}^{3} .
$$

Property 4: The dynamic terms $J(\cdot), C(\cdot), F(\cdot)$ satisfy the following bounds [12]

$$
\begin{aligned}
\|J(\varphi)-J(\phi)\|_{i \infty} & \leq \zeta_{j 1}\|\varphi-\phi\| \\
\left\|J^{-1}(\varphi)-J^{-1}(\phi)\right\|_{i \infty} & \leq \zeta_{j 2}\|\varphi-\phi\| \\
\|C(\varphi, \phi)\|_{i \infty} & \leq \zeta_{c 1}\|\phi\| \\
\|C(\varphi, \phi)-C(\kappa, \phi)\|_{i \infty} & \leq \zeta_{c 2}\|\phi\|\|\varphi-\kappa\| \\
\|F(\varphi)\|_{i \infty} & \leq \zeta_{f 1} \\
\|F(\varphi)-F(\phi)\|_{i \infty} & \leq \zeta_{f 2}\|\varphi-\phi\|
\end{aligned}
$$

\footnotetext{
${ }^{1}$ The entries of these dynamic terms are presented in Appendix I.
}

$\forall \varphi, \phi, \kappa \in \mathbb{R}^{3}, \zeta_{j 1}, \zeta_{j 2}, \zeta_{c 1}, \zeta_{c 2}, \zeta_{f 1}, \zeta_{f 2} \in \mathbb{R}$ are positive bounding constants, and $\|\cdot\|_{i \infty}$ denotes the induced infinity norm.

The mathematical model of the ship can be written in terms of the desired position and its time derivatives as follows

$$
W_{d} \triangleq J\left(\eta_{d}\right) \ddot{\eta}_{d}+C\left(\eta_{d}, \dot{\eta}_{d}\right) \dot{\eta}_{d}+F\left(\eta_{d}\right) \dot{\eta}_{d}
$$

where $W_{d}\left(\eta_{d}, \dot{\eta}_{d}, \ddot{\eta}_{d}\right) \in \mathbb{R}^{3}$ is a function of the desired position, velocity and acceleration, denoted by $\eta_{d}(t), \dot{\eta}_{d}(t)$, $\ddot{\eta}_{d}(t) \in \mathbb{R}^{3}$, respectively. In our analysis, we have made the common assumption that the desired position signal with its first three time derivatives are bounded functions of time.

\section{PROBlem Formulation}

Our control objective is to design a position tracking controller for the dynamically positioned ship model given by (5) when only the position of the ship $\eta(t)$ being available.

To quantify the tracking control objective, the position tracking error, denoted by $e(t) \in \mathbb{R}^{3}$, is defined as

$$
e \triangleq \eta_{d}-\eta
$$

And to compensate for the lack of velocity measurements, a velocity observation signal, denoted by $\hat{\hat{\eta}}(t) \in \mathbb{R}^{3}$, will be designed. The difference between the actual and observed versions of the velocity signal, the velocity observation error, denoted by $\dot{\widetilde{\eta}}(t) \in \mathbb{R}^{3}$, and a corresponding position observation error signal, denoted by $\widetilde{\eta}(t) \in \mathbb{R}^{3}$, are defined as

$$
\begin{aligned}
& \dot{\tilde{\eta}} \triangleq \dot{\eta}-\dot{\hat{\eta}} \\
& \tilde{\eta} \triangleq \eta-\widehat{\eta}
\end{aligned}
$$

where $\widehat{\eta}(t) \in \mathbb{R}^{3}$ is the observed position signal. In order to facilitate the subsequent stability analysis and to simplify the error system development, a filtered position tracking error, denoted by $r(t) \in \mathbb{R}^{3}$, and a filtered velocity observation error, denoted by $s(t) \in \mathbb{R}^{3}$, are constructed as

$$
\begin{aligned}
& r \triangleq \dot{e}+\alpha e \\
& s \triangleq \dot{\tilde{\eta}}+\alpha \widetilde{\eta}
\end{aligned}
$$

where $\alpha \in \mathbb{R}$ is a positive control gain.

\section{Control Design}

\section{A. Observer-controller couple design}

Based on the subsequent stability analysis, the velocity observer is designed as

$$
\dot{\hat{\eta}}=p+K_{0} \widetilde{\eta}-K_{c} e
$$

where $p(t) \in \mathbb{R}^{3}$ is an auxiliary filter signal updated according to

$$
\dot{p}=K_{1} \operatorname{Sgn}(\widetilde{\eta})+K_{2} \widetilde{\eta}-\alpha K_{c} e
$$

where $K_{0}, K_{c}, K_{1}, K_{2} \in \mathbb{R}^{3 \times 3}$ are diagonal, positivedefinite gain matrices, and $\operatorname{Sgn}(\cdot) \in \mathbb{R}^{3}$ is the vector signum 
function. Notice that, after utilizing (22) and (25), the time derivative of (24), can be obtained to have the following form

$$
\ddot{\hat{\eta}}=K_{1} \operatorname{Sgn}(\widetilde{\eta})+K_{2} \widetilde{\eta}+K_{0} \dot{\tilde{\eta}}-K_{c} r .
$$

The subsequent stability analysis enables us to design the control torque input vector $\tau^{*}(t)$ in the following form

$$
\tau^{*}=W_{d}+K_{p} e+\alpha K_{c}\left(\eta_{d}-\widehat{\eta}\right)+K_{c}\left(\dot{\eta}_{d}-\dot{\hat{\eta}}\right)
$$

where $K_{p} \in \mathbb{R}^{3 \times 3}$ is a diagonal, positive-definite control gain matrix.

It should be noted that, from the definitions in (19) and (21), following expression may be obtained

$$
\eta_{d}-\widehat{\eta}=e+\widetilde{\eta}
$$

Utilizing this and its time derivative, the control input of (27) can also be expressed in the following manner

$$
\tau^{*}=W_{d}+K_{p} e+K_{c}(r+s) .
$$

This formulation will be used to present the overall analysis in a more convenient way later in the stability analysis section.

\section{B. Observer analysis}

After utilizing (5) and (26) along with the time derivative of (20), we obtain the following formulation for the velocity observation error dynamics

$$
\begin{aligned}
\ddot{\widetilde{\eta}} & =\ddot{\eta}-\ddot{\widehat{\eta}} \\
& =N_{0}-K_{1} \operatorname{Sgn}(\widetilde{\eta})-K_{2} \tilde{\eta}-K_{0} \dot{\tilde{\eta}}+K_{c} r
\end{aligned}
$$

where $N_{0}(t) \in \mathbb{R}^{3}$ is an auxiliary term defined as

$$
N_{0} \triangleq J^{-1}\left(\tau^{*}-C \dot{\eta}-F \dot{\eta}\right)
$$

Substituting the control input signal of (29) and the definition of $W_{d}(\cdot)$ in (18) into (32), the auxiliary signal $N_{0}(t)$ can be partitioned as

$$
N_{0}=N_{d}+N_{b}
$$

where $N_{d}(t), N_{b}(t) \in \mathbb{R}^{3}$ are auxiliary terms defined as

$$
\begin{aligned}
N_{d} \triangleq & \ddot{\eta}_{d} \\
N_{b} \triangleq \quad & {\left[J^{-1}(\eta)-J^{-1}\left(\eta_{d}\right)\right] J\left(\eta_{d}\right) \ddot{\eta}_{d} } \\
& +J^{-1}(\eta)\left\{C\left(\eta_{d}, \dot{\eta}_{d}\right) \dot{\eta}_{d}-C(\eta, \dot{\eta}) \dot{\eta}\right. \\
& \left.+F\left(\eta_{d}\right) \dot{\eta}_{d}-F(\eta) \dot{\eta}+K_{p} e+K_{c}(r+s)\right\}
\end{aligned}
$$

Remark 1: Based on its definition in (35), the norm of $N_{b}(t)$ can be upper bounded as

$$
\left\|N_{b}\right\| \leq \rho_{01}\|e\|+\rho_{02}\|r\|+\rho_{03}\|e\|^{2}+\rho_{04}\|r\|^{2}+\rho_{05}\|s\|
$$

where $\rho_{01}, \rho_{02}, \rho_{03}, \rho_{04}, \rho_{05} \in \mathbb{R}$ are known positive bounding constants (see Appendix II for details).

After taking the time derivative of (23), the dynamics for the filtered observation error $s(t)$ is obtained as

$$
\dot{s}=N_{d}+N_{b}-K_{1} \operatorname{Sgn}(\widetilde{\eta})-K_{2} \widetilde{\eta}-K_{0} \dot{\tilde{\eta}}+K_{c} r+\alpha \dot{\tilde{\eta}}
$$

where (31) and (33) were utilized. After selecting the observer gains to satisfy

$$
\alpha\left(K_{0}-\alpha I_{3}\right)=K_{2}
$$

following expression can be obtained

$$
\dot{s}=N_{d}+N_{b}-K_{1} \operatorname{Sgn}(\widetilde{\eta})-\frac{K_{2}}{\alpha} s+K_{c} r
$$

where (23) was utilized.

Obtaining (39), we can state the following preliminary analysis. Consider the following non-negative scalar function, denoted by $V_{0}(t) \in \mathbb{R}$,

$$
V_{0} \triangleq \frac{1}{2} s^{T} s+P
$$

where $P(t) \in \mathbb{R}$ is an auxiliary non-negative function defined as

$$
P \triangleq \zeta_{P}-\int_{0}^{t} \omega(\sigma) d \sigma
$$

where $\omega(t), \zeta_{P} \in \mathbb{R}$ are defined as

$$
\begin{aligned}
\omega & \triangleq s^{T}\left(N_{d}-K_{1} \operatorname{Sgn}(\widetilde{\eta})\right) \\
\zeta_{P} & \triangleq \sum_{i=1}^{3} K_{1 i}\left|\widetilde{\eta}_{i}(0)\right|-\widetilde{\eta}^{T}(0) N_{d}(0) .
\end{aligned}
$$

As presented in [13], if $K_{1}$ is chosen to satisfy

$$
K_{1 i} \geq\left|N_{d i}(t)\right|+\frac{1}{\alpha}\left|\dot{N}_{d i}(t)\right|
$$

where $K_{1 i} \in \mathbb{R}$ denotes the $i$-th diagonal entry of $K_{1}$, and $N_{d i}(t), \dot{N}_{d i}(t)$ denote the $i$-th diagonal entries of $N_{d}(t)$, $\dot{N}_{d}(t)$, respectively, then $P(t)$ is non-negative. Given the non-negativeness of $P(t)$, it can be concluded that $V_{0}(t)$ is a Lyapunov function with respect to $\sqrt{P(t)}$ and $s(t)$. The time derivative of $V_{0}(t)$ can be obtained as

$$
\dot{V}_{0}=s^{T}\left(-\frac{1}{\alpha} K_{2} s+N_{b}+K_{c} r\right)
$$

where (39) and the time derivative of (41) were utilized.

\section{Error system development}

In order to obtain the dynamics for the filtered tracking error $r(t)$, we take its time derivative, pre-multiply with $J(\cdot)$, and after performing some straightforward algebraic manipulations, we reach

$$
J \dot{r}=-C r+W_{s}-\tau^{*}
$$

where $W_{s}(t) \in \mathbb{R}^{3}$ is an auxiliary term defined as

$$
W_{s}=J\left(\ddot{\eta}_{d}+\alpha \dot{e}\right)+C\left(\dot{\eta}_{d}+\alpha e\right)+F \dot{\eta} .
$$

Substituting the control input signal of (29) into (46), the following closed-loop error dynamics for $r(t)$ can be obtained

$$
J \dot{r}=-C r+\chi-K_{p} e-K_{c}(r+s)
$$

where $\chi(t) \in \mathbb{R}^{3}$ is an auxiliary error-like term defined as

$$
\chi \triangleq W_{s}-W_{d}
$$


Remark 2: Based on its definition in (49), the norm of the auxiliary term $\chi(t)$ can be upper bounded as

$$
\|\chi\| \leq \rho_{1}(\|e\|)\|e\|+\rho_{2}(\|e\|)\|r\|
$$

where $\rho_{1}(\|e\|), \rho_{2}(\|e\|) \in \mathbb{R}$ are known positive nondecreasing functions of their arguments (see Appendix II for details).

\section{Stability Analysis}

Theorem 1: The velocity observer in (24) and (25), and the control input signal of (27) ensure semi-global asymptotic stability of the closed-loop system in the sense that

$$
\|e(t)\|,\|\dot{\tilde{\eta}}(t)\| \rightarrow 0 \text { as } t \rightarrow+\infty
$$

provided that controller and observer gains are selected to satisfy (38), (44), and the controller gain $K_{c}$ and the observer gain $K_{2}$ are chosen as follows

$$
\begin{gathered}
K_{c}=\left(1+\rho_{2}+k_{n} \rho_{1}^{2}\right) I_{3} \\
K_{2}=\alpha\left(1+\rho_{05}+k_{n}\left(\rho_{01}^{2}+\rho_{02}^{2}+\rho_{03}^{2}+\rho_{04}^{2}\right)\right) I_{3}
\end{gathered}
$$

where $\rho_{1}(\|e\|), \rho_{2}(\|e\|)$ were introduced in (50), $\rho_{0 i}, i=$ $1, \ldots, 5$ were introduced in (36), and $k_{n} \in \mathbb{R}$ is a nonlinear damping gain selected to satisfy the following condition

$$
k_{n}>\frac{1}{2}+\frac{\lambda_{2}}{4 \lambda_{1}}\|z(0)\|^{2}
$$

and $z(t) \in \mathbb{R}^{10}$ is defined as

$$
z \triangleq\left[\begin{array}{llll}
\sqrt{P} & s^{T} & r^{T} & e^{T}
\end{array}\right]^{T}
$$

and the positive bounding constants $\lambda_{1}, \lambda_{2} \in \mathbb{R}$ are defined as

$$
\begin{aligned}
& \lambda_{1} \triangleq \frac{1}{2} \min \left\{1, J_{\min }, K_{p, \min }\right\} \\
& \lambda_{2} \triangleq \max \left\{1, \frac{J_{\max }}{2}, \frac{K_{p, \max }}{2}\right\}
\end{aligned}
$$

where subscripts min and max denote the minimum and maximum eigenvalues of a matrix, respectively.

Proof: A non-negative Lyapunov function, denoted by $V(z) \in \mathbb{R}$, is defined as

$$
V \triangleq V_{0}+\frac{1}{2} r^{T} J r+\frac{1}{2} e^{T} K_{p} e .
$$

The above Lyapunov function can be upper and lower bounded as

$$
\lambda_{1}\|x\|^{2} \leq \lambda_{1}\|z\|^{2} \leq V \leq \lambda_{2}\|z\|^{2}
$$

where $x(t) \in \mathbb{R}^{9}$ is defined as

$$
x \triangleq\left[\begin{array}{lll}
s^{T} & r^{T} & e^{T}
\end{array}\right]^{T} .
$$

The time derivative of $V(t)$ is obtained as

$$
\dot{V}=\dot{V}_{0}+r^{T} J \dot{r}+\frac{1}{2} r^{T} \dot{J} r+e^{T} K_{p} \dot{e}
$$

and after utilizing (10), (22), (45), (48), we obtain

$$
\dot{V}=s^{T} N_{b}-\frac{1}{\alpha} s^{T} K_{2} s+r^{T} \chi-r^{T} K_{c} r-\alpha e^{T} K_{p} e .
$$

After utilizing the upper bounds in (36) and (50), the righthand side of (62) can be upper bounded as

$$
\begin{aligned}
\dot{V} \leq & -\alpha K_{p, \min }\|e\|^{2}-\|r\|^{2}-\|s\|^{2} \\
& +\left[\rho_{01}\|e\|\|s\|-k_{n} \rho_{01}^{2}\|s\|^{2}\right] \\
& +\left[\rho_{02}\|r\|\|s\|-k_{n} \rho_{02}^{2}\|s\|^{2}\right] \\
& +\left[\rho_{03}\|e\|^{2}\|s\|-k_{n} \rho_{03}^{2}\|s\|^{2}\right] \\
& +\left[\rho_{04}\|r\|^{2}\|s\|-k_{n} \rho_{04}^{2}\|s\|^{2}\right] \\
& +\left[\rho_{1}\|e\|\|r\|-k_{n} \rho_{1}^{2}\|r\|^{2}\right]
\end{aligned}
$$

where (52) and (53) were utilized. After completing the squares of the bracketted terms, the right-hand side of (63) can be upper bounded as

$$
\begin{aligned}
\dot{V} \leq & -\left[\alpha K_{p, \min }-\frac{1}{2 k_{n}}-\frac{1}{4 k_{n}}\|e\|^{2}\right]\|e\|^{2} \\
& -\left[1-\frac{1}{4 k_{n}}-\frac{1}{4 k_{n}}\|r\|^{2}\right]\|r\|^{2}-\|s\|^{2} .
\end{aligned}
$$

Utilizing the definition of (60), the right-hand side of (64) can further be upper bounded to have the following form

$$
\dot{V} \leq-\left[1-\frac{1}{4 k_{n}}\left(2+\|x\|^{2}\right)\right]\|x\|^{2}
$$

where the gains are assumed to be chosen to satisfy $\alpha K_{p, \min } \geq 1$. The sign of the upper bound of $\dot{V}(t)$ is determined by the bracketted term in (65), and this term has to be non-negative to ensure the negative semi-definiteness of $\dot{V}(t)$. Mathematically speaking, we must have

$$
1-\frac{1}{4 k_{n}}\left(2+\|x\|^{2}\right)>0
$$

to ensure the negative semi-definiteness of $\dot{V}(t)$. A sufficient condition on (66) can be obtained as

$$
1-\frac{1}{4 k_{n}}\left(2+\frac{V}{\lambda_{1}}\right)>0
$$

where (59) was utilized, and hence the right-hand side of (65) can be reformulated as

$$
\dot{V} \leq-\beta\|x\|^{2} \text { provided that } 4 k_{n}>2+\frac{V}{\lambda_{1}}
$$

where $\beta \in \mathbb{R}$ is some positive constant satisfying $0<$ $\beta \leq 1$. Due to the negative semi-defineteness of $\dot{V}(t)$, the maximum value that $V(t)$ can have is its initial value, $V(0)$, therefore, after utilizing (59), a more conservative condition on $k_{n}$ can be obtained to have the following form

$$
\dot{V} \leq-\beta\|x\|^{2} \text { provided that } 4 k_{n}>2+\frac{\lambda_{2}}{\lambda_{1}}\|z(0)\|^{2}
$$

that is, when $k_{n}$ is selected to satisfy (54), we can ensure that $V(t)$ is bounded. Given the boundedness of $V(t)$, it is clear that $z(t) \in \mathcal{L}_{\infty}$, and thus, $e(t), r(t), s(t), P(t) \in \mathcal{L}_{\infty}$. After utilizing standard signal chasing arguments, we can show that all signals in the closed-loop system are bounded, and $e(t)$ and $\dot{\tilde{\eta}}(t)$ are uniformly continuous signals (from 
the boundedness of their time derivatives). Furthermore, after integrating both sides of (69), we can conclude that $x(t) \in \mathcal{L}_{2}$, and therefore $e(t), \dot{\tilde{\eta}}(t) \in \mathcal{L}_{2}$. Finally, utilizing Barbalat's Lemma [14], the asymptotic tracking result given in (51) can be obtained.

\section{NUMERICAL RESUlTS}

To illustrate the performance of the observer-controller couple, a numerical simulation with Matlab Simulink was performed. The ship model in (1) was utilized with the following inertia and damping matrices [3]

$$
\begin{gathered}
M=\left[\begin{array}{lll}
1.0852 & 0 & 0 \\
0 & 2.0575 & -0.4087 \\
0 & -0.4087 & 0.2153
\end{array}\right] \\
D=\left[\begin{array}{lll}
0.08656 & 0 & 0 \\
0 & 0.0762 & 0.1510 \\
0 & 0.0151 & 0.0031
\end{array}\right] .
\end{gathered}
$$

The desired position of the ship was given as [10]

$$
\eta_{d}=\left[\begin{array}{lll}
10 \sin (0.2 t) & 10 \cos (0.2 t) & 5 \sin (0.2 t)
\end{array}\right]^{T}
$$

with the initial positions $\eta(0)=\left[\begin{array}{lll}1 & -1 & 1\end{array}\right]^{T}$, and the initial velocities $\dot{\eta}(0)$ were set to zero. Controller and observer gains were tuned via a trial-and-error method until a good tracking performance was achieved, and were chosen as

$$
\begin{aligned}
& K_{0}=\operatorname{diag}\left\{\begin{array}{lll}
15 & 20 & 7.5
\end{array}\right\} \\
& K_{1}=\operatorname{diag}\left\{\begin{array}{lll}
0.75 & 0.15 & 0.15
\end{array}\right\} \\
& K_{c}=0.5 I_{3}, K_{p}=I_{3}, \alpha=2.5 .
\end{aligned}
$$

The results are shown in Figures 1-4. In Figure 1, the actual position $\eta(t)$ and the desired position $\eta_{d}(t)$ were presented. In Figures 2 and 3, the position tracking error $e(t)$ and the control input torque $\tau^{*}(t)$ were presented, respectively. In Figure 4, the position observation error $\tilde{\eta}(t)$ was presented. From Figures 1 and 2, it is clear that the tracking control objective was met.
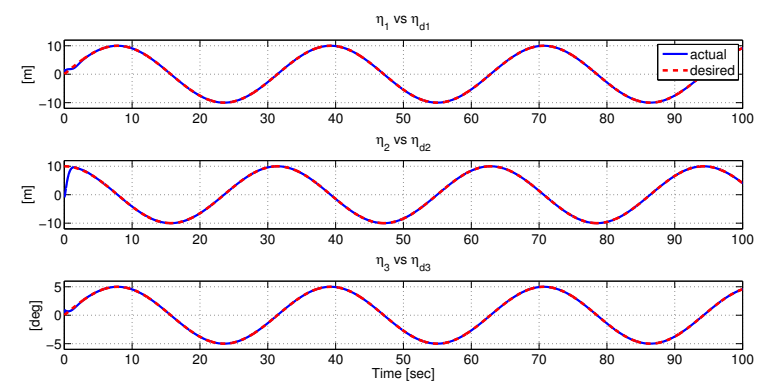

Fig. 1. The desired position $\eta_{d}(t)$ (dotted) and the actual position $\eta(t)$ (solid)
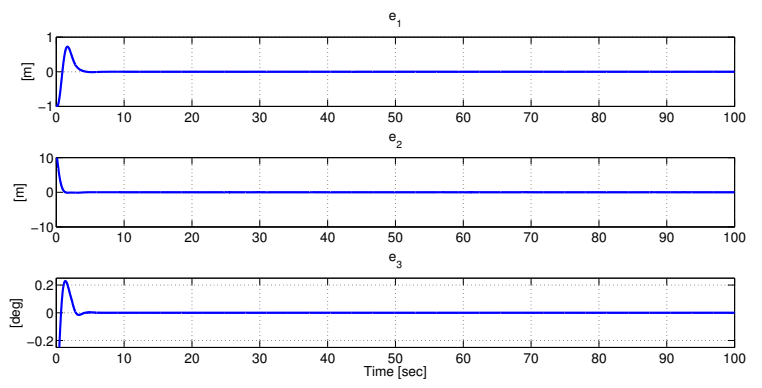

Fig. 2. The tracking error $e(t)$

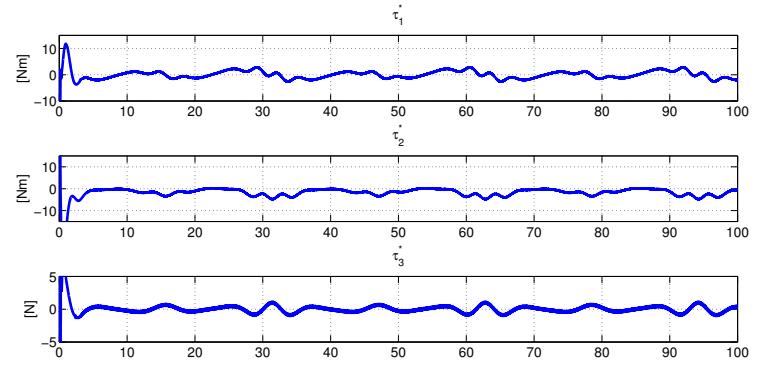

Fig. 3. Control input torque $\tau^{*}(t)$

\section{CONCLUSION}

In this paper, we have presented a new observer formulation for dynamically positioned surface vessels. A novel observer-controller formulation, backed up with a Lyapunov-type analysis has been presented. Our formulation achieved semi-global tracking despite the lack of velocity measurements. Simulation results were presented to illustrate the tracking performance of the observer-controller couple.

In its current form, the proposed methodology requires the exact knowledge of the system parameters. However it is our sincere belief that with considerably small effort adaptive, robust, and repetitive learning versions of the same observercontroller structure can be designed to compensate for the parametric uncertainty, thus future work will focus on dealing with structured and unstructured uncertainties of the overall system.

\section{APPENDIX I \\ DYNAMIC TERMS}

The dynamic terms $J(\eta), C(\eta, \dot{\eta})$ and $F(\eta)$ defined in (6) and (7) are calculated as follows

$$
\begin{gathered}
J=\left[\begin{array}{lll}
m_{11} c_{\psi}^{2}+m_{22} s_{\psi}^{2} & \left(m_{11}-m_{22}\right) s_{\psi} c_{\psi} & -m_{23} s_{\psi} \\
\left(m_{11}-m_{22}\right) s_{\psi} c_{\psi} & m_{11} s_{\psi}^{2}+m_{22} c_{\psi}^{2} & m_{23} c_{\psi} \\
-m_{23} s_{\psi} & m_{23} c_{\psi} & m_{33}
\end{array}\right] \\
C=\dot{\psi}\left[\begin{array}{lll}
\left(m_{22}-m_{11}\right) s_{\psi} c_{\psi} & m_{11} c_{\psi}^{2}+m_{22} s_{\psi}^{2} & 0 \\
-m_{11} s_{\psi}^{2}-m_{22} c_{\psi}^{2} & \left(m_{11}-m_{22}\right) s_{\psi} c_{\psi} & 0 \\
-m_{23} c_{\psi} & -m_{23} s_{\psi} & 0
\end{array}\right] \\
F=\left[\begin{array}{lll}
d_{11} c_{\psi}^{2}+d_{22} s_{\psi}^{2} & \left(d_{11}-d_{22}\right) s_{\psi} c_{\psi} & -d_{23} s_{\psi} \\
\left(d_{11}-d_{22}\right) s_{\psi} c_{\psi} & d_{11} s_{\psi}^{2}+d_{22} c_{\psi}^{2} & d_{23} c_{\psi} \\
-d_{32} s_{\psi} & d_{32} c_{\psi} & d_{33}
\end{array}\right] .
\end{gathered}
$$



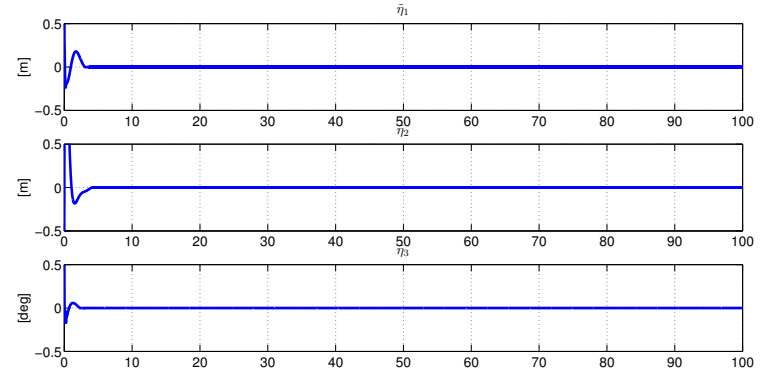

Fig. 4. Position observation error $\widetilde{\eta}(t)$

\section{APPENDIX II \\ PROOF OF BOUNDS}

In this appendix, the upper bounds of the norm of $N_{b}(t)$ in (36) and the norm of $\chi(t)$ in (50) will be obtained. Specifically, after utilizing (8), (9), (11), (13), (14), (15), (16), (17), along with (35), we can obtain

$$
\begin{aligned}
\left\|N_{b}\right\| \leq & \frac{1}{m_{l}}\left\{\zeta_{j 2} m_{u} m_{l}\left\|\ddot{\eta}_{d}\right\|+\zeta_{c 2}\left\|\dot{\eta}_{d}\right\|^{2}+\alpha \zeta_{c 1}\left\|\dot{\eta}_{d}\right\|\right. \\
& \left.+\alpha \zeta_{f 1}+\zeta_{f 2}\left\|\dot{\eta}_{d}\right\|+K_{p, \max }\right\}\|e\| \\
& +\frac{1}{m_{l}}\left\{2 \zeta_{c 1}\left\|\dot{\eta}_{d}\right\|+\zeta_{f 1}+K_{c, \max }\right\}\|r\| \\
& +\frac{2 \alpha^{2} \zeta_{c 1}}{m_{l}}\|e\|^{2}+\frac{2 \zeta_{c 1}}{m_{l}}\|r\|^{2}+\frac{K_{c, \max }}{m_{l}}\|s\|(74)
\end{aligned}
$$

where $2 \alpha\|e\|\|r\| \leq \alpha^{2}\|e\|^{2}+\|r\|^{2}$ and $\|\dot{e}\| \leq \alpha\|e\|+\|r\|$ were utilized. From the structure of (74), it is clear that the bounding constants $\rho_{0 i}, i=1, \ldots, 5$ can be defined as

$$
\begin{aligned}
\rho_{01} \triangleq & \frac{1}{m_{l}}\left\{\zeta_{j 2} m_{u} m_{l}\left\|\ddot{\eta}_{d}\right\|+\zeta_{c 2}\left\|\dot{\eta}_{d}\right\|^{2}+\alpha \zeta_{c 1}\left\|\dot{\eta}_{d}\right\|\right. \\
& \left.+\alpha \zeta_{f 1}+\zeta_{f 2}\left\|\dot{\eta}_{d}\right\|+K_{p, \max }\right\} \\
\rho_{02} \triangleq & \frac{1}{m_{l}}\left\{2 \zeta_{c 1}\left\|\dot{\eta}_{d}\right\|+\zeta_{f 1}+K_{c, \max }\right\} \\
\rho_{03} \triangleq & \frac{2 \alpha^{2} \zeta_{c 1}}{m_{l}}, \rho_{04} \triangleq \frac{2 \zeta_{c 1}}{m_{l}}, \rho_{05} \triangleq \frac{K_{c, \max }}{m_{l}}
\end{aligned}
$$

to obtain the upper bound of the norm of $N_{b}(t)$ in (36).

After subsituting the definitions of $W_{d}(t)$ and $W_{s}(t)$ in (18) and (47), respectively, into the definition of $\chi(t)$ in (49), we obtain

$$
\begin{aligned}
\|\chi\| \leq & \left\{\alpha^{2} m_{u}+\zeta_{j 1}\left\|\ddot{\eta}_{d}\right\|+2 \alpha \zeta_{c 1}\left\|\dot{\eta}_{d}\right\|+\zeta_{f 2}\left\|\dot{\eta}_{d}\right\|\right. \\
& \left.+\alpha \zeta_{f 1}+\zeta_{c 2}\left\|\dot{\eta}_{d}\right\|^{2}+\alpha^{2} \zeta_{c 1}\|e\|\right\}\|e\| \\
& +\left\{\alpha m_{u}+\zeta_{c 1}\left\|\dot{\eta}_{d}\right\|+\alpha \zeta_{c 1}\|e\|\right\}\|r\|
\end{aligned}
$$

where (8), (11), (12), (14), (15), (16), (17) were utilized. When the bounding functions $\rho_{1}(e)$ and $\rho_{2}(e)$ are selected as

$$
\begin{aligned}
\rho_{1}(e) \triangleq & \alpha^{2} m_{u}+\zeta_{j 1}\left\|\ddot{\eta}_{d}\right\|+2 \alpha \zeta_{c 1}\left\|\dot{\eta}_{d}\right\|+\zeta_{f 2}\left\|\dot{\eta}_{d}\right\| \\
& +\alpha \zeta_{f 1}+\zeta_{c 2}\left\|\dot{\eta}_{d}\right\|^{2}+\alpha^{2} \zeta_{c 1}\|e\| \\
\rho_{2}(e) \triangleq & \alpha m_{u}+\zeta_{c 1}\left\|\dot{\eta}_{d}\right\|+\alpha \zeta_{c 1}\|e\|
\end{aligned}
$$

\section{REFERENCES}

[1] T. I. Fossen, Marine Control Systems: Guidance, Navigation, and Control of Ships, Rigs and Underwater Vehicles. Trondheim, Norway: Marine Cybernetics AS, 2002.

[2] A. Loria, T. I. Fossen, and E. Panteley, "A separation principle for dynamic positioning of ships: Theoretical and experimental results," IEEE Trans. Contr. Syst. Technol., vol. 8, no. 2, pp. 322-343, 2000.

[3] T. I. Fossen and ^. Grøvlen, "Nonlinear output feedback control of dynamically positioned ships using vectorial observer backstepping," IEEE Trans. Contr. Syst. Technol., vol. 6, no. 1, pp. 121-128, 1998.

[4] J. G. Balchen, N. A. Jenssen, and S. Sællid, "Dynamic positioning of floating vessels based on kalman filtering and optimal control," in Proc. IEEE Int. Conf. Decision and Control, Albuquerque, NM, USA, 1980, pp. 852-864.

[5] - "Dynamic positioning using kalman filtering and optimal control theory," in Proc. of IFAC/IFIP Symp. on Aut. in Offshore Oil Field Operation, Holland, Amsterdam, 1976, pp. 183-186.

[6] M. J. Grimble, R. J. Patton, and D. A. Wise, "The design of dynamic positioning control systems using stochastic optimal control theory," Optimal Control Applications \& Methods, vol. 1, pp. 167-202, 1980

[7] A. J. Sørensen, S. I. Sagatun, and T. I. Fossen, "Design of a dynamic positioning system using model-based control," Control Engineering Practice, vol. 4, no. 3, pp. 359-368, 1996.

[8] O. Fjellstad and T. I. Fossen, "Quaternion feedback regulation of underwater vehicles," in Proc. of IEEE Int. Conf. on Control Applications, Glasgow, Scotland, 1994, pp. 857-862.

[9] C. C. D. Wit, E. Olguin, D. Perrier, and M. Perrier, "Robust nonlinear control of an underwater vehicle/manipulator system with composite dynamics," in Proc. IEEE Int. Conf. Robot. Autom., Leuven, Belgium, 1998, pp. 452-457.

[10] Y. Fang, E. Zergeroglu, M. S. de Queiroz, and D. M. Dawson, "Global output feedback control of dynamically positioned surface vessels: An adaptive control approach," Mechatronics, vol. 14, no. 4, pp. 341-356, 2004.

[11] M. Wondergem, E. Lefeber, K. Y. Pettersen, and H. Nijmeijer, "Output feedback tracking of ships," IEEE Trans. Contr. Syst. Technol., vol. 19, no. 2, pp. 442-448, 2011.

[12] W. E. Dixon, A. Behal, D. M. Dawson, and S. Nagarkatti, Nonlinear Control of Engineering Systems: A Lyapunov-Based Approach. Boston, MA, USA: Birkhauser, 2003.

[13] B. Xian, D. M. Dawson, M. S. de Queiroz, and J. Chen, "A continuous asymptotic tracking control strategy for uncertain nonlinear systems," IEEE Trans. Automat. Contr, vol. 49, no. 7, pp. 1206-1211, 2004.

[14] M. Krstic, I. Kanellakopoulos, and P. Kokotovic, Nonlinear and Adaptive Control Design. New York, NY, USA: John Wiley and Sons, 1995.

then the bound given in (50) is obtained. 